\title{
Intergeracionalidade das Habilidades Sociais entre Pais e Filhos Adolescentes
}

\author{
Camila Negreiros Comodo ${ }^{1}$ \\ Instituto de Terapia por Contingências de Reforçamento (ITCR) \\ Almir Del Prette \\ Zilda Aparecida Pereira Del Prette \\ Universidade Federal de São Carlos
}

\begin{abstract}
RESUMO - Pesquisas sobre genitores e filhos têm apontado resultados no sentido da transmissão intergeracional de comportamentos. Considerando que as habilidades sociais são vistas como um fator de proteção para adolescentes, o presente estudo teve por objetivo identificar a possível transmissão do repertório de habilidades sociais de pais e mães para filhos adolescentes. Uma amostra de 142 adolescentes e seus genitores foi avaliada por meio dos inventários IHS-Del-Prette e IHSA-Del-Prette, procedendo-se às análises estatísticas. Os resultados encontrados sugeriram a transmissão de assertividade, empatia, abordagem afetiva e desenvoltura social, a depender do sexo dos genitores e dos filhos, assim como da idade dos adolescentes. Foram discutidas a importância do repertório elaborado de habilidades sociais nos genitores e as implicações dos resultados encontrados.
\end{abstract}

Palavras-chave: habilidades sociais, intergeracionalidade, relações entre gerações, adolescência

\section{Intergenarational Transmission of Social Skills between Parentes and Adolecenst Children}

\begin{abstract}
Research on parents and children have pointed out results on intergenerational transmission of behaviors. Considering that social skills are considered as a protective factor against deficits and disorders in adolescence, the present study aimed to identify the possible transmission of social skills between parents and their adolescent children. IHS-Del-Prette and IHSA-Del-Prette inventories were administered to a sample of 142 adolescents and their parents, after which statistical analysis were carried out. Results suggested the transmission of assertiveness, empathy, affective approach and social resourcefulness, depending on the gender of parents and children and on the age of the adolescents. The importance of the social skills repertoire in parents was discussed, as well as the implications of the findings.
\end{abstract}

Keywords: social skills, intergenerational transmission, intergenerational relations, adolescence

Diversos estudos (Bowers \& Yehuda, 2016; Hopkins, Olson, Pain, \& Vincett, 2011; Leve, Khurana, \& Reich, 2015), principalmente no exterior, têm investigado a questão da transmissão intergeracional de padrões de comportamentos. Porém, na área de habilidades sociais, essa linha de pesquisa ainda se mostra incipiente. Considerando que as habilidades sociais são comportamentos aprendidos na interação com agentes de socialização (Cia, Pereira, Ruas, Del Prette, \& Del Prette, 2006; Gresham, 2009), o presente estudo discute aspectos teóricos e empíricos relativos à transmissão intergeracional das habilidades sociais de genitores para filhos adolescentes.

\section{Transmissão Intergeracional de Comportamentos}

A transmissão de comportamentos pode ser entendida, genericamente, como o conjunto das diferentes formas pelas quais os indivíduos aprendem com outros membros do seu grupo social, com os quais convivem, os padrões

1 Endereço para correspondência: Rua Jasmim, 612, Apto 84, Torre 2, Chácara Primavera, Campinas, São Paulo, Brasil. CEP: 13087-460.

E-mail:cami_nc@hotmail.com de comportamento que estes apresentam (Baum, 2004). A partir disso, consideram-se importantes as diferentes relações sociais estabelecidas ao longo da vida, em especial a relação entre genitores e filhos. Nessa direção, estudos teóricos e empíricos têm sido realizados com o objetivo de verificar comportamentos dos pais e mães e de seus descendentes, buscando explicar por que e como os comportamentos dos filhos reproduzem ou divergem dos de seus genitores (Shaffer, Burt, Obradovic, Herbes, \& Masten, 2009). Nos Estados Unidos e na Europa, uma vertente das pesquisas sobre esse fenômeno adotou o nome de intergeracionalidade ou transmissão intergeracional, referindo-se à repetição de padrões de comportamento ao longo das gerações (p. ex., Bowers \& Yehuda, 2016; Hopkins et al., 2011; Leve et al., 2015).

A transmissão intergeracional de comportamentos é um tema que vem ganhando a atenção de pesquisadores de várias disciplinas, incluindo a Psicologia e a Sociologia. As pesquisas nessa área realizadas apenas com um informante como fonte de dados (ou filhos ou pais) encontraram intergeracionalidade, por exemplo, da violência intrafamiliar (Kim, 2009), do baixo autocontrole (Boutwell \& Beaver, 2010), de transtornos de ansiedade (Eley et al., 2015), do uso 
de tabaco (Escario \& Wilkinson, 2015), da prática de maustratos com os filhos (Leve et al., 2015) e do estresse (Bowers \& Yehuda, 2016). Nessas pesquisas, apenas os filhos ou os genitores foram os informantes, tomando-se apenas o relato de uma parte da díade. Os estudos realizados com mais de um informante encontraram transmissão intergeracional de comportamentos de preservação do meio ambiente, como separar o lixo reciclável e economizar energia (Grønhøj \& Thøgersen, 2009), de racismo (Duriez \& Soenens, 2009), de participação política (Quintelier, 2015), de práticas parentais (Madden et al., 2015), de formação religiosa (Hopkins et al., 2011) e do comportamento de dirigir (Miller \& TabmanBem-Ari, 2010).

No Brasil, as pesquisas sobre transmissão intergeracional de comportamentos ainda são incipientes. Os estudos localizados sobre esse tema apontam a transmissão de violência conjugal (Paixão et al., 2015), de estilos (Oliveira et al., 2002; Weber, Selig, Bernardi, \& Salvador, 2006) e práticas parentais (Marin et al.,, 2013), do comportamento moral (Weber \& Gomide, 2007), da obesidade (Almeida \& Netto Junior, 2015) e da anorexia nervosa (Valdanha, Scorsolini-Comin, \& Santos, 2013).

Alguns estudos, tanto nacionais quanto estrangeiros, denotam uma visão de intergeracionalidade apenas no sentido de repetição de aspectos de um padrão relacional pais-filhos, que as crianças e adolescentes adotariam na interação com seus filhos no futuro (Madden, et al., 2015; Marin et al., 2013; Weber, Selig, Bernardi, \& Salvador, 2006). Entretanto, outros estudos apontam que o conceito de transmissão intergeracional é mais amplo e inclui a possível transmissão de qualquer comportamento individual (p. ex., Almeida \& Netto Junior, 2015; Quintelier, 2015).

Cabe também destacar, a partir da análise desses estudos, a possibilidade da coleta de dados com dois informantes (tanto os genitores quanto os filhos) como uma forma de garantir maior confiabilidade das informações obtidas, uma vez que cada membro da díade pode relatar sobre o próprio comportamento, sem a necessidade de relatos de terceiros. É fundamental enfatizar ainda que, não obstante todos os estudos referidos apresentem resultados em direção à intergeracionalidade, existem também diferenças nos padrões de comportamentos de genitores e filhos. Os filhos podem ter repertórios diferentes de seus genitores por participarem de outros grupos sociais, alguns dos quais reproduzem os comportamentos de seus pais e mães e outros não (Sabatier \& Lannegrand, 2005; Weber et al., 2006).

\section{Habilidades Sociais}

A intergeracionalidade pode ocorrer para os mais diversos comportamentos, sejam eles motores ou sociais. As habilidades sociais constituem um conjunto especial de comportamentos sociais com alta probabilidade de produzir consequências positivas imediatas ou mediatas tanto para um indivíduo como para as pessoas do seu grupo social (Del Prette \& Del Prette, 2010). Del Prette e Del Prette (2011) estabelecem uma diferença entre os conceitos de habilidades sociais e de competência social. Essa última tem sido definida como "atributo avaliativo de um comportamento ou conjunto de comportamentos bem sucedidos - conforme determinados critérios de funcionalidade - em uma interação social" (Del Prette \& Del Prette, 2010, p. 106). Os critérios de funcionalidade se referem tanto à consecução de objetivos imediatos na interação social, incluindo a aprovação do desempenho pela comunidade verbal quanto a consequências de médio e longo prazo para o indivíduo e seu grupo social (Del Prette \& Del Prette, 2011).

As investigações com a população de adolescentes mostram que o repertório de habilidades sociais vem sendo correlacionado com um desempenho escolar satisfatório (Feitosa, Del Prette, \& Del Prette, 2012) e maior aceitação pelos pares (Gonçalves \& Murta, 2008). Por outro lado, um repertório pobre em habilidades sociais nessa faixa etária está correlacionado com problemas de comportamento (CasaliRobalinho, Del Prette, \& Del Prette, 2015), dificuldade de aprendizagem (Cook et al., 2008) e transtornos diversos, como depressão (Campos, Del Prette, \& Del Prette, 2014), abuso de substâncias (Sá \& Del Prette, 2014) e transtornos alimentares (Uzunian \& Vitalle, 2015). A adolescência é um período importante para o refinamento de habilidades sociais aprendidas na infância e para o estabelecimento e manutenção de interações sociais saudáveis (Murta, Del Prette, Nunes, \& Del Prette, 2006). A exacerbação de problemas que atingem os jovens na cultura ocidental contemporânea, como gravidez precoce, evasão escolar e aliciamento para atividades ilegais, como também a presença de problemas psicológicos como bullying, alcoolismo, drogadição, depressão e timidez (Reis, de Almeida, Miranda, Alves, \& Madeira, 2013), tem gerado uma preocupação crescente com relação ao desenvolvimento socioemocional e sua interface com a saúde nessa fase do desenvolvimento.

Dada a importância de habilidades sociais no repertório dos indivíduos, os pesquisadores têm se voltado para as condições que favorecem a aprendizagem e o aperfeiçoamento desses comportamentos, especialmente nos ambientes mais críticos para adolescentes, que são a família e a escola (Del Prette \& Del Prette, 2005). Pais, professores e pares ensinam habilidades sociais e outros comportamentos, sejam desejáveis ou não, principalmente por meio de três estratégias principais: o manejo de consequências, a instrução e a modelação (Gresham, 2009).

Para o presente estudo, a estratégia da modelação é particularmente importante, uma vez que a replicação de padrões de comportamentos entre as gerações supõe imitação dos comportamentos dos genitores pelos filhos. Skinner (1953/2007) afirma que o comportamento a ser imitado funciona como estímulo discriminativo, indicando que, se o indivíduo se comportar da mesma maneira, provavelmente produzirá as mesmas consequências reforçadoras. De acordo com Baum (2004), há três condições importantes para a imitação. A primeira seria a frequência com que os comportamentos ocorrem na sociedade, ou seja, quando um comportamento é mais disseminado, maior a probabilidade de que outros venham a imitá-lo. Em segundo lugar, é mais provável a imitação dos modelos que são frequentemente vistos pela pessoa, como pais, amigos, pessoas famosas etc. A terceira, mas não menos importante, é a efetividade do comportamento: a imitação é mais provável para comportamentos bem-sucedidos, ou seja, que são 
reforçados. Nesse sentido, Baum afirma que a ocorrência do comportamento não depende apenas da presença de modelos, mas também das consequências que determinada resposta produz na sua interação com o ambiente.

Considerando o papel dos pais na transmissão de padrões de comportamento a seus filhos e a importância do repertório de habilidades sociais para os jovens entende-se como defensável investigar como esses comportamentos sociais são (ou não) replicados na segunda geração. Portanto, este estudo teve como objetivo analisar a possível correlação entre o repertório de habilidades sociais de pais e filhos, tomando-a como indicador de transmissão intergeracional na família.

\section{Método}

\section{Participantes e Local}

Participaram do estudo 142 adolescentes entre 12 e 17 anos (43\% homens e $57 \%$ mulheres), os quais responderam ao IHSA-Del-Prette (Del Prette \& Del Prette, 2009). Também participaram 131 mães (com idade entre 29 e 59 anos, média: 41,$6 ; d p: 5,95$ ) e 131 pais do sexo masculino (com idade entre 20 e 66 anos, média 45,02; $d p: 7,526)$, os quais responderam ao IHS-Del Prette (2001). De acordo com as normas do manual do IHSA-Del-Prette (2009b), os adolescentes da amostra foram divididos em dois grupos de acordo com a idade: (a) de 12 a 14 anos (50,7\% da amostra) e (b) de 15 a 17 anos (49,3\% da amostra). Tanto os adolescentes quanto os pais responderam aos instrumentos em salas e horários reservados pelas escolas dos filhos. Convém esclarecer que, para cada adolescente participante, tanto o pai quanto a mãe respondiam ao questionário em questão, porém, o número de pais de cada sexo é menor que o número total de adolescentes, pois houve 11 casos em que os adolescentes eram irmãos e por isso foram utilizados os dados dos mesmos pais.

$\mathrm{Na}$ busca de uma amostra heterogênea, a pesquisa foi realizada tanto em escolas públicas $(48,6 \%$ dos respondentes) quanto privadas (51,4\% dos respondentes). As escolas participantes da pesquisa foram selecionadas por conveniência, e os critérios para a participação na pesquisa foram o consentimento de pais e adolescentes, assim como a localização dos filhos na faixa etária de 12 a 17 anos.

\section{Instrumentos}

Inventário de Habilidades Sociais - IHS-Del-Prette (Del Prette \& Del Prette, 2001). Trata-se de um instrumento tipo lápis-papel, de autorrelato, validado para o contexto brasileiro, que contém 38 questões, cada uma descrevendo uma situação de demanda de desempenho social e uma possível reação a ela. Solicita-se que o respondente estime a frequência com que reage da forma sugerida em cada item com base em uma escala tipo Likert que varia de zero (nunca ou raramente) até quatro (sempre ou quase sempre). Na apuração, é produzido um escore geral e cinco escores associados aos fatores: (F1) Enfrentamento e autoafirmação com risco; (F2) Expressão de sentimento positivo; (F3) Conversação e desenvoltura social; (F4) Autoexposição a desconhecidos e situações novas; e (F5) Autocontrole da agressividade. $\mathrm{O}$ instrumento apresenta indicadores de confiabilidade e consistência interna (Alfa de Cronbach $=0,75$; estabilidade teste-reteste: $r=0,90 ; p=0,001)$ e validade concomitante com o Inventário de Rathus $(r=0,79$; $p=0,01)$.

Inventário de Habilidades Sociais para Adolescentes - IHSA-Del-Prette (Del Prette \& Del Prette, 2009). Instrumento de autorrelato tipo lápis-papel validado para o contexto brasileiro. Contém 38 itens, sendo que cada um apresenta uma situação interpessoal e um desempenho social e o adolescente é solicitado a responder indicando: (a) quão difícil é para ele apresentar a reação indicada no item e (b) qual a frequência com que apresenta a reação indicada em cada item. Nesses dois indicadores (frequência e dificuldade), as respostas são mensuradas em uma escala tipo Likert. Para a frequência, as categorias de respostas são as mesmas do IHS-Del-Prette e, para a dificuldade, a escala varia entre nenhuma e muita dificuldade. Para a presente pesquisa, considerando os objetivos, somente a escala de frequência foi utilizada. $\mathrm{Na}$ apuração é produzido um escore geral e seis escores para os fatores: (F1) Empatia; (F2) Autocontrole; (F3) Civilidade; (F4) Assertividade; (F5) Abordagem afetiva; e (F6) Desenvoltura social. O instrumento apresenta indicadores de confiabilidade e de consistência interna (Alfa de Cronbach $=0,896$; estabilidade teste-reteste: $r=0,844, p<0,001)$.

\section{Procedimentos Éticos e de Coleta de Dados}

A pesquisa foi aprovada pelo Comitê de Ética (CEP/ UFSCar, parecer $\left.n^{\circ}=222 / 2010\right)$. Com o consentimento dos pais, dos adolescentes e a permissão das escolas para a realização da coleta de dados em suas dependências, a primeira autora aplicou os instrumentos em grupo junto aos adolescentes e individualmente junto aos pais.

\section{Tratamento de Dados}

Os dados obtidos com os inventários foram computados sob a forma de escores de acordo com as instruções dos manuais e organizados em planilhas do SPSS 16.0 (Statistical Package for the Social Sciences), procedendo-se às análises estatísticas descritivas (média, desvio-padrão) e inferenciais (correlação de Spearman), adotando-se o nível de significância de $p<0,05$. A amostra foi analisada inicialmente por meio do teste de Mann-Whitney em termos de suas características demográficas e foram verificadas diferenças nas habilidades sociais dos pais a depender do sexo (pais com maiores escores em autoafirmação com risco, desenvoltura social autoexposição a desconhecidos e autocontrole em relação às mães; e mães com escores mais elevados em expressão de sentimento positivo do que os pais) e dos filhos a depender do sexo (filhas com maiores escores em empatia e civilidade em relação aos filhos), nas habilidades sociais das adolescentes do sexo feminino a depender da faixa etária (filhas mais velhas com escores maiores em autocontrole 
em detrimento das filhas mais novas) e nas habilidades sociais dos adolescentes do sexo masculino a depender do tipo de escola (filhos que estudavam em escolas particulares tiveram escores maiores em empatia, autocontrole, civilidade e desenvoltura social em relação a filhos que estudavam em escolas públicas ${ }^{1}$. Como foram identificadas essas diferenças no repertório social dos adolescentes e dos pais segundo características sociodemográficas, foram efetuadas análises de correlação diferenciadas para cada uma dessas subamostras.

A hipótese de transmissão intergeracional está baseada, no presente estudo, na correlação entre os fatores do IHSDel-Prette (Del Prette \& Del Prette, 2001) e os fatores do IHSA-Del-Prette (Del Prette \& Del Prette, 2009). Segundo o manual do IHSA-Del Prette esse instrumento foi elaborado com base no IHS-Del Prette, tendo modificações apenas com relação aos interlocutores, contextos e demandas próprios de cada faixa etária. Sendo assim, a presente pesquisa
Assertividade. Em relação aos pais, a intergeracionalidade ocorreu para a habilidade de Assertividade.

Quanto aos adolescentes do sexo masculino, as correlações investigadas diferiram para o grupo de pais e o grupo de mães. Com as mães, as análises foram subdivididas em tipos de escolas e, com os pais, subdivididas por tipo de escola e idade dos filhos. A Tabela 3 mostra apenas as correlações significativas entre os escores de habilidades sociais dos genitores e dos filhos de escola particular.

Chama a atenção, na Tabela 3 , o número maior de correlações - e todas negativas - entre escores dos pais e dos filhos do que entre mães e filhos, indicando que quanto melhor o desempenho do pai, pior o desempenho do filho nas classes de habilidades sociais indicadas. Em outras palavras, não há dados sugestivos de transmissão intergeracional de pais para filhos na subamostra de escola particular. Em relação à mãe, as duas correlações significativas podem ser tomadas como indicadores de transmissão intergeracional: habilidades de Expressão de sentimentos e Autoexposição a

Tabela 1. Similaridades funcionais entre os escores do IHS-Del-Prette e do IHSA-Del-Prette.

IHS-Del-Prette
Escore Total
(F1) Enfrentamento e autoafirmação com risco
(F2) Expressão de sentimento positivo
(F3) Conversação e desenvoltura social
(F4) Autoexposição a desconhecidos e situações novase Total
(F5) Autocontrole da agressividade.

considera similaridade funcional entre as subescalas dos dois instrumentos, como pode ser observado na Tabela 1 .

\section{Resultados}

$\mathrm{Na}$ análise dos dados das filhas mais velhas (15 a 17 anos) e de seus genitores não foi encontrada nenhuma correlação entre os escores das adolescentes e os escores de seus pais ${ }^{2}$, e apenas uma correlação entre o Fator 3, Conversação $e$ desenvoltura social, da mãe e o Fator 2, Autocontrole, da filha $(\rho=0,352 ; p=0,028)$. A Tabela 2 , a seguir, mostra somente as correlações significativas encontradas entre o repertório de habilidades sociais de filhas mais novas (12 a 14 anos) e de seus genitores.

$\mathrm{Na}$ tabela, é possível notar um maior número de correlações entre as habilidades sociais das filhas e das mães, do que das filhas e de seus pais. Considerando a possível intergeracionalidade, em relação às mães, ela ocorreu para as habilidades sociais de Empatia e também para a

1 Para maiores detalhes acerca das análises de comparação entre os repertórios de habilidades sociais dos participantes da presente pesquisa, consultar a dissertação da primeira autora.

2 No decorrer do presente estudo, o termo pais está associado aos genitores do sexo masculino desconhecidos (mães) correlacionadas com as habilidades de Abordagem afetiva (filhos).

Com relação aos adolescentes do sexo masculino que frequentavam escolas públicas, houve somente uma correlação com o repertório de habilidades sociais de seus pais: habilidades de Autoafirmação na expressão de sentimento positivo dos pais e habilidades de Civilidade dos filhos $(\rho=0,361 ; p=0,050)$. No que se refere às mães, foi observada uma correlação positiva entre habilidades de Autoexposição a desconhecidos da mãe e habilidades de Desenvoltura social do filho $(\rho=0,406 ; p=0,026)$, denotando intergeracionalidade, e uma correlação negativa entre habilidades de Autocontrole da agressividade da mãe e de Desenvoltura social do filho $(\rho=-0,368 ; p=0,045)$, mas que não foi tomada como indicador de interacionalidade conforme explicitado na Tabela 1.

Nas análises das habilidades sociais dos pais e dos adolescentes do sexo masculino mais novos (12 a 14 anos), não foi encontrada nenhuma correlação. Para os adolescentes mais velhos (15 a 17 anos), as correlações significativas entre o repertório dos pais e dos filhos podem ser observadas na Tabela 4.

A Tabela 4 mostra uma única correlação positiva e indicativa de transmissão intergeracional entre pais e filhos, no caso de filhos mais velhos, associando habilidades de Expressão de sentimentos positivos dos pais com habilidades 
Tabela 2. Correlações (Spearman) entre escores de habilidades sociais dos genitores e das filhas de 12 a 14 anos (sombreadas as correlações tomadas como possiveis indicadores de intergeracionalidade).

\begin{tabular}{llll}
\hline \multicolumn{1}{c}{ Fatores dos genitores } & \multicolumn{1}{c}{ Fatores das filhas } & $\boldsymbol{\rho}$ & $\boldsymbol{p}$ \\
\hline F2 mãe (Expressão de sentimento positivo) & Escore geral filha & 0,461 & $0,002^{*}$ \\
F5 mãe (Autocontrole) & Escore geral filha & $-0,308$ & $0,048^{*}$ \\
F2 mãe (Expressão de sentimento positivo) & F1 filha (Empatia) & 0,385 & $0,012^{*}$ \\
F2 mãe (Expressão de sentimento positivo) & F3 filha (Civilidade) & 0,345 & $0,025^{*}$ \\
F5 mãe (Autocontrole) & F3 filha (Civilidade) & $-0,382$ & $0,013^{*}$ \\
F1 mãe (Autoafirmação com risco) & F4 filha (Assertividade) & 0,344 & $0,026^{*}$ \\
Escore geral pai & F4 filha (Assertividade) & 0,323 & $0,037^{*}$ \\
F1 pai (Autoafirmação com risco) & F4 filha (Assertividade) & 0,390 & $0,011^{*}$ \\
F2 pai (Expressão de sentimento positivo) & F6 filha (Desenvoltura social) & 0,354 & $0,021^{*}$ \\
\hline
\end{tabular}

Nota. ${ }^{*} p \leq 0,05$.

Tabela 3. Correlações (Spearman) entre escores de habilidades sociais dos genitores e dos filhos de escola particular (sombreadas as correlações tomadas como possíveis indicadores de intergeracionalidade).

\begin{tabular}{llll}
\hline \multicolumn{1}{c}{ Fatores dos genitores } & \multicolumn{1}{c}{ Fatores das filhos } & $\boldsymbol{\rho}$ & $\boldsymbol{p}$ \\
\hline F2 mãe (Expressão de sentimento positivo) & F5 filho (Abordagem afetiva) & 0,364 & $0,044^{*}$ \\
F4 mãe (Autoexposição a desconhecidos) & F5 filho (Abordagem afetiva) & 0,433 & $0,015^{*}$ \\
F5 mãe (Autocontrole) & F2 filho (Autocontrole) & $-0,385$ & $0,032^{*}$ \\
F5 mãe (Autocontrole) & F6 filho (Desenvoltura social) & $-0,507$ & $0,004^{*}$ \\
Escore geral pai & Escore geral filho & $-0,442$ & $0,013^{*}$ \\
Escore geral pai & F2 filho (Autocontrole) & $-0,570$ & $0,001^{*}$ \\
F3 pai (Conversação e desenvoltura social) & F1 filho (Empatia) & $-0,480$ & $0,006^{*}$ \\
F3 pai (Conversação e desenvoltura social) & Escore geral filho & $-0,555$ & $0,001^{*}$ \\
F3 pai (Conversação e desenvoltura social) & F2 filho (Autocontrole) & $-0,639$ & $0,000^{*}$ \\
F3 pai (Civilidade) & F6 filho (Desenvoltura social) & $-0,394$ & $0,028^{*}$ \\
F4 pai (Autoexposição a desconhecidos) & F6 filho (Desenvoltura social) & $-0,371$ & $0,040^{*}$ \\
\hline Nota *p<0,05. & & &
\end{tabular}
Nota. ${ }^{*} p \leq 0,05$.

Tabela 4. Correlações (Spearman) entre escores de habilidades sociais dos pais e dos filhos de 15 a 17 anos (sombreadas as correlações tomadas como possiveis indicadores de intergeracionalidade).

\begin{tabular}{llll}
\hline \multicolumn{1}{c}{ Fatores dos pais } & \multicolumn{1}{c}{ Fatores das filhas } & $\boldsymbol{\rho}$ & $\boldsymbol{p}$ \\
\hline F2 (Expressão de sentimento positivo) & Escore geral filho & 0,387 & $0,032^{*}$ \\
F2 (Expressão de sentimento positivo) & F1 filho (Empatia) & 0,405 & $0,024^{*}$ \\
F2 (Expressão de sentimento positivo) & F3 filho (Civilidade) & 0,403 & $0,025^{*}$ \\
F2 (Expressão de sentimento positivo) & F4 filho (Assertividade) & 0,378 & $0,036^{*}$ \\
F2 (Expressão de sentimento positivo) & F6 filho (Desenvoltura social) & 0,423 & $0,018^{*}$ \\
F3 (Conversação e desenvoltura social) & F6 filho (Desenvoltura social) & $-0,356$ & $0,049^{*}$ \\
\hline Nota. ${ }^{*}$ p $\leq 0,05$. & & &
\end{tabular}

de Empatia dos filhos. Um resumo das correlações indicativas de transmissão intergeracional, obtidas neste estudo, é apresentado na Tabela 5 .

Do conjunto de 10 possibilidades de correlações indicativas de transmissão intergeracional (portanto positivas), o presente estudo encontrou seis, conforme indicadas na Tabela 5 (uma delas encontrada duas vezes - de pais para filhas e de mães para filhas). Nessa Tabela, também se observa que, das sete correlações indicativas de intergeracionalidade encontradas, cinco se referem ao repertório de mães para com o repertório dos seus filhos e apenas duas apontam relação entre o repertório de pais e seus filhos. Para as adolescentes do sexo feminino de 12 a 14 anos, pode-se ressaltar os resultados na direção da transmissão intergeracional, de mãe para filha, das habilidades assertivas e empáticas. Nas correlações entre pais e filhas mais novas, pode-se observar a possível transmissão intergeracional da assertividade. Para os adolescentes do sexo masculino e suas mães, ressalta-se a provável transmissão intergeracional das habilidades sociais de abordagem afetiva para os filhos 
Tabela 5. Síntese dos possiveis indicadores de transmissão intergeracional nas correlações encontradas no presente estudo

\begin{tabular}{|c|c|c|}
\hline IHS-Del-Prette & IHSA-Del-Prette & Correlações encontradas \\
\hline F1-Enfrentamento e autoafirmação com risco & F4-Assertividade & Mães e filhas mais novas \\
\hline F1-Enfrentamento e autoafirmação com risco & F4-Assertividade & $\begin{array}{l}\text { Mães e filhas mais novas Pais } \\
\text { e filhas mais novas }\end{array}$ \\
\hline F2-Expressão de sentimento positivo & F1-Empatia & Mães e filhas mais novas \\
\hline F2-Expressão de sentimento positivo & F5-Abordagem afetiva & $\begin{array}{l}\text { Mães e filhos que frequentavam escolas } \\
\text { particulares }\end{array}$ \\
\hline F2-Expressão de sentimento positivo & F1-Empatia & Pais e filhos mais velhos \\
\hline F4-Autoexposição a desconhecidos e situações novas & F5- Abordagem afetiva & $\begin{array}{l}\text { Mães e filhos que frequentavam escolas } \\
\text { particulares }\end{array}$ \\
\hline F4-Autoexposição a desconhecidos e situações novas & F6-Desenvoltura social & $\begin{array}{l}\text { Mães e filhos que frequentavam escolas } \\
\text { públicas }\end{array}$ \\
\hline
\end{tabular}

Nota. ${ }^{*} p \leq 0,05$.

de escola particular e a transmissão de desenvoltura social para os de escola pública. Por fim, para os adolescentes do sexo masculino de 15 a 17 anos e seus pais, foi encontrada uma correlação indicativa de transmissão intergeracional, na classe de habilidades de empatia.

\section{Discussão}

A análise de correlação dos escores de habilidades sociais dos genitores e dos filhos adolescentes mostrou alguns resultados na direção da transmissão intergeracional desses comportamentos e outros indicando ausência de transmissão ou até mesmo um efeito contrário, em direção oposta a essa transmissão. Os dados apontam para mais correlações entre habilidades sociais de adolescentes e suas mães do que em relação a seus pais. Esse dado contraria, em parte, a concepção, encontrada em alguns estudos (Miller \& TabmanBem-Ari, 2010; Weber \& Gomide, 2007), de que os filhos imitam comportamentos do genitor do mesmo sexo. Uma possível explicação para os dados encontrados neste estudo poderia estar relacionada com a maior responsabilidade atribuída à mãe na educação dos filhos na cultura ocidental (Silveira, Pacheco, Schneider, \& Cruz, 2005). Essa hipótese é coerente com o pressuposto (Baum, 2004) de que a imitação é mais provável quando o comportamento é frequente na sociedade e o modelo é frequentemente visto pela pessoa. Nesse sentido, a mãe tem mais oportunidades de fornecer modelos de comportamento para os filhos, de ambos os sexos do que o pai. Ainda considerando a responsabilidade das genitoras mães pela educação dos filhos, é possível que elas tenham mais oportunidades de reforçar os comportamentos de seus filhos, sendo que elas provavelmente reforçam comportamentos que apresentam e que valorizam (Baum, 2004).

$\mathrm{Na}$ amostra de adolescentes do sexo feminino, a transmissão de habilidades sociais somente foi inferida nas correlações entre escores dos genitores e as adolescentes mais novas. Considerando que os indivíduos tendem a imitar modelos bem-sucedidos e frequentemente vistos (Baum, 2004) e que a adolescência é o período em que se passa a interagir mais tempo com os pares sem a supervisão de adultos (Cole \& Cole, 2004), é possível que, principalmente no final dessa fase do desenvolvimento, os pares sejam os principais agentes de reforço, punição, instrução e modelação. Isso poderia explicar a falta de correlações entre o repertório social de genitores e de suas filhas mais velhas. Em outras palavras, o avanço da idade supõe, em nossa cultura, maior autonomia e, também, maiores oportunidades de participação em novos grupos sociais, de forma independente dos pais e mães. Não obstante esse processo, os resultados sugestivos de transmissão de empatia entre pais e filhos do sexo masculino de 15 a 17 anos indicam que os pais continuam a influenciar os filhos nessa etapa do desenvolvimento.

Adicionalmente, o tipo de escola, aqui tomado como um indicador de condição socioeconômica em nosso meio, parece influenciar a transmissão de habilidades sociais dos adolescentes do sexo masculino. As habilidades sociais de abordagem afetiva seriam mais provavelmente transmitidas de mães de classe média ou média alta para seus filhos e a desenvoltura social teria maior probabilidade de ser transmitida de mães de classes menos favorecidas para seus filhos. Embora haja diferenças entre essas duas classes de habilidades sociais, sendo a abordagem afetiva mais relacionada a relações íntimas e a desenvoltura social relacionada a qualquer interação social, pode-se afirmar que as duas classes envolvem a exposição social e requerem habilidades de conversação, o que diminui o impacto dos tipos de escola nessa transmissão.

As correlações verificadas entre o repertório social de genitores e filhos mostram que a classe de expressão de sentimentos positivos nos genitores foi a mais amplamente correlacionada com os escores de diversas classes de habilidades sociais dos filhos. A habilidade dos pais e das mães de expressar sentimentos positivos parece ser necessária para que estes reforcem comportamentos apropriados dos filhos, por meio de elogios, carinhos e atenção. Dessa forma, trata-se de uma classe de habilidades sociais que está estreitamente vinculada com as habilidades sociais educativas, principalmente com a monitoria positiva, que envolve habilidades como elogiar, apresentar feedback positivo e demonstrar empatia (Del Prette \& Del Prette, 2008). Portanto, esse repertório social dos genitores pode ser importante não somente para transmitir tais comportamentos 
para os filhos por meio de modelação, mas também para que esses agentes sociais consigam ensinar comportamentos para os adolescentes por meio de consequenciação e instrução.

Correlações negativas entre o repertório social dos genitores e dos filhos também foram encontradas. Esses dados sugerem que, não obstante a transmissão de alguns comportamentos de genitores para filhos, os adolescentes também se comportam em oposição ao que observam de seus genitores (Amin, Lundborg, \& Rooth, 2015; Sabatier \& Lannegrand, 2005; Weber et al., 2006). É provável que os adolescentes apresentem determinados comportamentos reforçados pela família e outros comportamentos, concorrentes, reforçados pelos pares, e que estes acabem se tornando modelos mais fortes a seguir, principalmente nessa fase do desenvolvimento (Cole \& Cole, 2004). Podese exemplificar aqui os estudos descritos por Cole e Cole (2004), que apontam maior probabilidade de os adolescentes se engajarem em comportamentos similares aos de seus pares, como o consumo de bebidas alcoólicas e cigarro.

No que se refere a essa descontinuidade de comportamentos entre genitores e filhos, os escores dos adolescentes do sexo masculino da presente pesquisa apresentam mais correlações negativas com os de seus genitores do que as adolescentes mulheres, podendo indicar que esses adolescentes têm uma tendência maior a imitar outros padrões de comportamento que não aqueles emitidos por seus pais e mães. Nessa direção, Cole e Cole (2004) afirmam que os filhos, mais do que as filhas, nessa idade, estariam preocupados em estabelecer sua independência em relação aos genitores e outros adultos. Em contrapartida, uma análise mais detalhada desses dados mostra que algumas correlações negativas possuem particularidades.

No caso das mães, todas as correlações negativas entre seu repertório social e o de seus filhos envolviam a classe de habilidades sociais de Autocontrole da agressividade das mães, que teve uma média de frequência baixa nessa amostra $(0,703)$. Isso significa que os filhos da presente pesquisa têm um bom repertório de autocontrole, não obstante o repertório deficitário de autocontrole das mães. É possível que os filhos não vejam as mães como um modelo bem-sucedido com relação a esse comportamento e passem a imitar outros modelos que não elas, o que vai na direção da explicação de Baum (2004) de que um dos critérios para a imitação envolve o quanto o comportamento foi bem-sucedido. No caso dos pais a maioria das correlações negativas encontradas envolvia a classe de Conversação e desenvoltura social dos mesmos, indicando que o comportamento de conversação dos filhos parece ser mais influenciado por outros agentes sociais, como os pares, os quais são mais frequentemente vistos pelos adolescentes e podem ser entendidos como mais bem-sucedidos.

Cabe mencionar que a grande maioria das correlações encontradas foi de magnitude baixa ou média, o que significa que a força da relação entre as variáveis independente (escores dos genitores) e dependente (escores dos filhos) é apenas média (Cozby, 2003). Essa observação é importante, pois não se pode desconsiderar que, além das interações com a família, o repertório social dos adolescentes é produto também de sua interação com outros agentes sociais, como professores e pares (Del Prette \& Del Prette, 2005; Hopkins et al., 2011). O dado sugere, portanto, cautela em relação à hipótese de intergeracionalidade e um desafio de pesquisa: a dificuldade de separar os padrões de comportamentos transmitidos pela família dos demais comportamentos apresentados pelo adolescente em suas interações.

Nesse sentido, não se pode ignorar o papel da escola em promover habilidades sociais. Del Prette e Del Prette (2005) afirmam que, ao frequentar a escola, as crianças e adolescentes ampliam a gama de interlocutores, de demandas sociais e de oportunidades para aperfeiçoar seu repertório de habilidades sociais. Professores e pares se constituem em fonte de instrução, reforço, punição e modelação. Na adolescência, em especial, os pares assumem um papel ainda mais importante, pois, como destacam Novak e Peláz (2004), "pares fornecem uma fonte de experiências positivas para adolescentes porque eles ajudam o desenvolvimento das habilidades sociais dos adolescentes por meio de reforçamento positivo" (p. 474). Além de proverem reforço positivo, os pares também funcionam como modelos a serem seguidos (Martin \& Pear, 2009).

De todo modo, os resultados encontrados sugerem três implicações importantes. Primeiro, as classes de habilidades sociais em que foram verificadas a transmissão intergeracional - assertividade, empatia, abordagem afetiva e desenvoltura social - poderiam ser privilegiadas em programas de desenvolvimento e aprimoramento de habilidades sociais, voltados aos genitores. Segundo, programas para pais e mães, com o objetivo de modificar comportamentos nos filhos, poderiam ser realizados não apenas no sentido de promover as habilidades sociais educativas desses agentes, como pode ser visto nos programas de treinamento para essa população (Del Prette, Rocha, \& Del Prette, 2011; Orti, Bolsoni-Silva, \& Bratifish, 2015), mas também direcionados para habilidades sociais gerais dos genitores. Terceiro, entendendo-se que as habilidades sociais são comportamentos que podem ser transmitidos ao longo das gerações, pode ser importante investigar o papel dessas habilidades nas práticas culturais, assumindo-se, sob a perspectiva da Análise do Comportamento (Dittrich, 2004; Skinner, 1953/2007), que as habilidades sociais estariam possivelmente envolvidas em práticas culturais benéficas para a sobrevivência dos grupos sociais e da cultura (Del Prette \& Del Prette, 2010). Essa implicação é coerente com a posição de Glenn (1986), que defende uma reconstrução gradual de práticas desejáveis, a partir de iniciativas junto a pequenos grupos (como o grupo familiar, por exemplo), justificando a promoção e manutenção de comportamentos nessa direção.

\section{Considerações Finais}

O presente estudo traz algumas evidências na direção da replicação de padrões de comportamentos sociais entre genitores e filhos. No entanto, as correlações foram de magnitude baixa ou média, sugerindo influência de outros agentes sociais sobre as habilidades sociais desses adolescentes, como pares e professores, e também, outras estratégias de aprendizagem para além dos modelos.

Uma vez que a maioria dos estudos acerca da transmissão intergeracional de comportamentos utilizou respostas apenas 
dos filhos como informantes ou não teve como participantes ambos os genitores, pode-se afirmar que esta pesquisa trouxe uma contribuição para a melhor compreensão desses processos. No entanto, diversas questões ainda ficam em aberto para serem investigadas, evidenciando as limitações de estudos correlacionais. Em termos de alternativas metodológicas, entendem-se como importantes os estudos de díades com e sem bom repertório de habilidades sociais, bem como pesquisas longitudinais, que possam acompanhar especialmente a transição da infância para a adolescência. Estudos desse tipo poderiam, ainda, comparar a influência dos genitores com a de outros agentes de socialização na aprendizagem de habilidades sociais dos jovens.

São reconhecidas algumas limitações do presente estudo. Em termos de generalização dos achados, seria importante contar com amostras maiores de participantes em estudos futuros. Uma limitação adicional foi a utilização de apenas uma forma de avaliação do repertório social da amostra, o que restringe o objeto pesquisado às habilidades sociais e impede generalizações em termos de competência social (Del Prette \& Del Prette, 2005). Ainda considerando os instrumentos utilizados, cabe também ressaltar que o IHSDel Prette (Del Prette \& Del Prette, 2001) foi validado para o contexto brasileiro junto a uma população universitária, a qual pode ter características distintas da população de genitores da presente amostra. Dadas as limitações próprias dos instrumentos de relato (Del Prette \& Del Prette, 1999), outros instrumentos de medida como observação e entrevistas poderiam ser utilizados em estudos futuros, em articulação ou como alternativa aos inventários.

\section{Referências}

Almeida, A. T. C., \& Netto Junior, J. L. S. (2015). Measures of intergenerational transmission of obesity in Brazil. Ciência e Saúde Coletiva, 20(5), 1400-1413.

Amin, V., Lundborg, P., \& Rooth, D. O. (2015). The intergenerational transmission of schooling: Are mothers really less important than fathers? Economics of Education Review, 47, 100-117.

Baum, W. M. (2004). Understanding behaviorism: Behavior, culture and evolution. Wiley-Blackwell.

Boutwell, B. B., \& Beaver, K. M. (2010). The intergenerational transmission of low self-control. Journal of Research in Crime and Delinquency, 47(2), 174-209.

Bowers, M. E., \& Yehuda, R. (2016). Intergenerational transmission of stress in humans. Neuropsychopharmacology, 41(1), 232244.

Campos, J. R., Del Prette, A., \& Del Prette Z. A. P. (2014). Depressão na adolescência: Habilidades sociais e variáveis sociodemográficas como fatores de risco/proteção. Estudos e Pesquisas em Psicologia, 14(2), 408-428.

Casali-Robalinho, I. G., Del Prette, Z. A. P., \& Del Prette, A. (2015). Habilidades sociais como preditoras de problemas de comportamento em escolares. Psicologia: Teoria e Pesquisa, 31, 321-330.

Cia, F., Pereira, C. S., Ruas, T. C., Del Prette, Z. A. P., \& Del Prette, A. (2006). Habilidades sociais parentais e relacionamento entre pais e filhos. Psicologia em Estudo, 11(1), 73-81.
Cole, M., \& Cole S. R. (2004). O desenvolvimento da criança e do adolescente. Porto Alegre: Artmed.

Cook, C. R., Gresham, F. M., Kern, L., Barreras, R. B., Thornton, S., \& Crews, S. D. (2008). Social skills training with secondary EBD students: A review and analysis of meta-analytic literature. Journal of Emotional and Behavioral Disorders, 16, 131-144.

Cozby, P. C. (2003). Métodos de pesquisa em ciências do comportamento. São Paulo: Atlas.

Del Prette, A., \& Del Prette, Z. A. P. (2009). Inventário de Habilidades Sociais para Adolescentes (IHSA-Del Prette): Manual de aplicação, apuração e interpretação. São Paulo: Casa do Psicólogo.

Del Prette, A., \& Del Prette, Z.A.P. (2011). Enfoques e modelos do treinamento de habilidades sociais. In A. Del Prette \& Z.A.P. Del Prette (Orgs), Habilidades sociais: Intervenções efetivas em grupo (pp. 19-56). São Paulo: Casa do Psicólogo.

Del Prette, Z. A. P., \& Del Prette, A. (1999). Psicologia das habilidades sociais: Terapia, educação e trabalho. Petrópolis: Vozes.

Del Prette, Z. A. P., \& Del Prette, A. (2001). Inventário de Habilidades Sociais (IHS-Del Prette): Manual de aplicação, apuração e interpretação. São Paulo: Casa do Psicólogo.

Del Prette, Z. A. P., \& Del Prette, A. (2005). Psicologia das habilidades sociais na infância: Teoria e prática. Petrópolis: Vozes.

Del Prette, Z. A. P., \& Del Prette, A. (2008). Um sistema de categorias de habilidades sociais educativas. Paideia, 18(41), 517-530.

Del Prette Z. A. P., \& Del Prette, A. (2010) Habilidades sociais e análise do comportamento: Proximidade histórica e atualidades. Perspectivas em Análise do Comportamento, 1(2), 104-115.

Del Prette, Z. A. P., Rocha, M. M., \& Del Prette, A. (2011). Habilidades sociais na infância: Avaliação e intervenção com a criança e seus pais. In C. S. Petersen \& R. Wainer (Orgs), Terapias cognitivo-comportamentais para crianças e adolescentes (pp. 46-61). Porto Alegre: Artmed.

Dittrich, A. (2004). Behaviorismo radical, ética e politica: Aspectos teóricos do compromisso social (Tese de doutorado não publicada). Centro de Educação e Ciências Humanas, Universidade Federal de São Carlos, São Carlos, São Paulo, Brasil.

Duriez, B., \& Soenens, B. (2009). The intergenerational transmission of racism: The role of right-wing authoritarianism and social dominance orientation. Journal of Research in Personality, 43, 906-909.

Eley, T. C., McAdams, T. A., Rijsdijk, F. V., Lichtenstein, P., Narusyte, J., Reiss, D., Spotts, E. L., ... Neiderhiser, J. M. (2015). The Intergenerational transmission of anxiety: A children-of-twins study. American Journal of Psychiatry, 172(7), 630-637.

Escario, J. J., \& Wilkinson, A. V. (2015). The intergenerational transmission of smoking across three cohabitant generations: A count data approach. Journal of Community Health, 40(5), 912-919.

Feitosa, F. B., Del Prette, Z. A. P., \& Del Prette, A. (2012). Social skills and academic achievement: The mediating function of cognitive competence. Temas em Psicologia, 20(1), 61-70.

Glenn, S. (1986) Metacontingencies in Walden Two. Behavior Analysis and Social Action, 5, 2-8. 
Gonçalves, E. S., \& Murta, S. G. (2008). Avaliação dos efeitos de uma modalidade de treinamento de habilidades sociais para crianças. Psicologia Reflexão e Crítica, 21(3), 430-436.

Gresham, F. M. (2009). Análise do comportamento aplicada às habilidades sociais. In Z. A. P. Del Prette \& A. Del Prette (Orgs), Psicologia das Habilidades Sociais: Diversidade teórica e suas implicações (pp. 17-66). Petrópolis, RJ: Vozes.

Grønhøj, A., \& Thøgersen, J. (2009). Like father, like son? Intergenerational transmission of values, attitudes and behaviours in the environmental domain. Journal of Environmental Psychology, 29, 414-421.

Hopkins, P., Olson, E., Pain, R., \& Vincett, G. (2011). Mapping intergenerationalities: The formation of youthful religiosities. Transactions of the Institute of British Geographers, 36(2), 314-327.

Kim, J. (2009). Type-specific intergenerational transmission of neglectful and physically abusive parents behaviors among young parents. Children and Youth Services Review, 31, 761767.

Leve, L. D., Khurana, A., \& Reich, E. B. (2015). Intergenerational transmission of maltreatment: A multilevel examination. Development and Psychopathology, 27(4), 1429-1442.

Madden, V., Domoney, J., Aumayer, K., Sethna, V., Iles, J., Hubbard, I., ... Ramchandani, P. (2015). Intergenerational transmission of parenting: Findings from a UK longitudinal study. European Journal of Public Health, 25(6), 1030-1035.

Marin, A. H., Martins, G. F., Freitas, A. P. C. O., Silva, I. M., Lopes, R. C. S., \& Piccinini, C. A. (2013). Transmissão intergeracional de práticas educativas parentais: Evidências empíricas. Psicologia: Teoria e Pesquisa, 29(2), 123-132.

Martin, G., \& Pear, J. (2009). Modificação do comportamento: $O$ que é e como fazer (N. C. de Aguirre, Trad., $8^{\mathrm{a}}$ Ed.). São Paulo: Roca.

Miller, G., \& Taubman-Ben-Ari, O. (2010). Driving styles among young novice drivers: The contribution of parental driving styles and personal characteristics. Accident Analysis and Prevention, 42, 558-570.

Murta, S. G., Del Prette, A., Nunes, F.C., \& Del Prette, Z. A. P. (2006). Problemas en la adolescencia: Contribuciones del entrenamiento en habilidades sociales In J. C. Salamanca (Ed.), Manual de intervención psicológica para adolescentes: Ámbito de la salud y educativo (Unidade 1, Cap. 2). Bogotá: PSICOM Editores.

Novak, G., \& Peláz, M. (2004). Child and adolescent development: A behavioral system approach. Thousand Oaks, California: Sage Publications.
Oliveira, E. A., Marin, A. H., Pires, F. B., Frizzo, G. B., Ravanello, T., \& Rossato, C. (2002). Estilos parentais autoritário e democrático-recíproco intergeracionais, conflito conjugal e comportamentos de externalização e internalização. Psicologia: Reflexão e Crítica, 15(1), 1-11.

Orti, N. P., Bolsoni-Silva, A. T., \& Bratifish, M. (2015). Assessment of the effects of a parental intervention with mothers of children with internalizing problems. Advances in Research, 4, 279-292.

Paixão, G. P. N., Gomes, N. P., Diniz, N. M. F., Lira, M. O. S. C., Carvalho, M. R. S., \& Silva, R. S. (2015). Women experiencing the intergenerationality of conjugal violence. Revista LatinoAmericana de Enfermagem, 23(5), 874-879.

Quintelier, E. (2015). Intergenerational transmission of political participation intention. Acta Politica, 50(3), 279-296.

Reis, D. C., de Almeida, T. A. C., Miranda, M. M., Alves, R. H., \& Madeira, A. M. F. (2013). Vulnerabilidades à saúde na adolescência: Condições socioeconômicas, redes sociais, drogas e violência. Revista Latino Americana de Enfermagem, 21(2), 586-594.

Sá, L. G. C., \& Del Prette, Z. A. P. (2014). Habilidades sociais como preditoras do envolvimento com álcool e outras drogas: Um estudo exploratório. Interação em Psicologia (Online), 18(2), 167-178.

Sabatier, C., \& Lannegrand-Willems, L. (2005). Transmission of family values and attachment: A French tree-generation study. Applied Psychology: An International Review, 54(3), 378-395.

Shaffer, A., Burth, K. B., Obradovic, J., Herbers, J. E., \& Masten, A. S. (2009). Intergenerational continuity in parenting quality: The mediating role of social competence. Developmental Psychology, 45(5), 1227- 1240.

Silveira, L. M. O. B., Pacheco, J. T. B., Schneider, A. A., \& Cruz, T. (2005). Estratégias educativas desejáveis e indesejáveis: Uma comparação entre a percepção de pais e de mães de adolescentes. Alethéia, 21, 31-42.

Skinner, B. F. (1953/2007). Ciência e comportamento humano. (J. C. Todorov e R. Azzi, Trad.). São Paulo: Martins Fontes.

Uzunian, L. G., \& Vitalle, M. S. S. (2015). Habilidades sociais: Fator de proteção contra transtornos alimentares em adolescentes. Ciência e saúde coletiva, 20(11), 3495-3508.

Valdanha, E. D., Scorsolini-Comin, F., \& Santos, M. A. (2013). Anorexia nervosa e transmissão psíquica transgeracional. Revista Latinoamericana de Psicopatologia Fundamental, 16(1), 71-88.

Weber, L. N. D., Selig, G. A., Bernardi, M. G., \& Salvador, A. P. V. (2006). Continuidade dos estilos parentais através das gerações: Transmissão intergeracional de estilos parentais. Paidéia, 16(35), 407-414.

Weber, L., \& Gomide, P. I. C. (2007). Relação entre comportamento moral dos pais e dos filhos adolescentes. Estudos de Psicologia, 24(1), 53-60. 